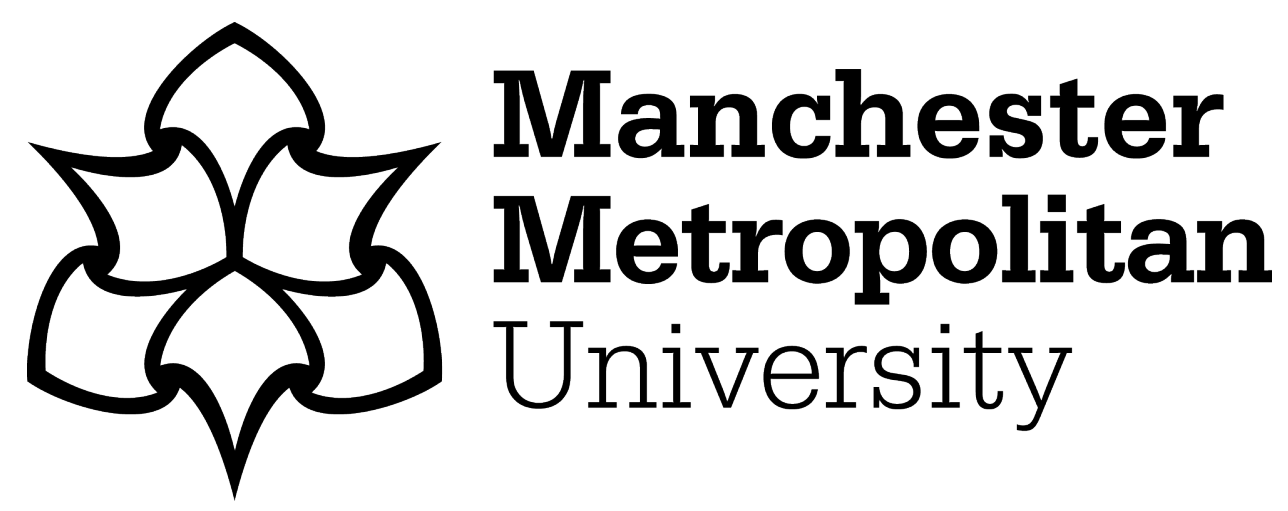

Yamamoto, S, Liskiewicz, T ORCID logoORCID: https://orcid.org/0000-00020866-814X, Fujimura, K, Tashiro, K and Takai, O (2018) Temperature rise of diamond-like carbon during sliding: Consideration of the real contact area. Tribology International, 131. pp. 496-507. ISSN 0301-679X

Downloaded from: https://e-space.mmu.ac.uk/624360/

Version: Accepted Version

Publisher: Elsevier BV

DOI: https://doi.org/10.1016/j.triboint.2018.09.022

Please cite the published version 


\title{
TEMPERATURE RISE OF DIAMOND-LIKE CARBON DURING SLIDING: CONSIDERATION OF THE REAL CONTACT AREA
}

\author{
S. Yamamoto ${ }^{1 *}$, T. Liskiewicz ${ }^{2}$, K. Fujimura ${ }^{1}$, K. Tashiro ${ }^{1}$, O. Takai $^{1}$ \\ ${ }^{1}$ Kanto Gakuin University, Materials and Surface Laboratory, Japan \\ ${ }^{2}$ University of Leeds, School of Mechanical Engineering, UK
}

\begin{abstract}
The real contact area based on modified Hertzian theory derived from sinusoidal asperity model is introduced and the measurement method for the real contact area is proposed. The real contact approach is compared with the Greenwood-Williamson theory showing good agreement between the two methods. The separation between the contact surfaces is estimated by taking into account the surface roughness. The temperature rise at the sliding interface between the DLC coating and E52100 steel is evaluated experimentally by the thermal indicator paint method, and the real contact area ratio is estimated from the temperature simulation data. The temperature rise is discussed from the entropy balance point of view induced from the real contact area and the frictional energy.
\end{abstract}

Keywords: Sliding; Roughness; Contact temperature; Contact area 


\section{Nomenclature}

A $\quad=$ individual contact area

An = apparent contact area

$\mathrm{Ar} \quad=$ real contact area

a $\quad=$ contact radius

$\mathrm{d}=$ distance between the two surfaces

$\mathrm{d}_{0} \quad=$ normalized $\mathrm{d}$

$\mathrm{E} \quad=$ Youngs' modulus

E' = equivalent modules of elasticity

$\mathrm{H}(\mathrm{x}) \quad=$ entropy of the normal distribution in the probability theory

$\mathrm{h} \quad=$ asperity height

$\mathrm{m}_{3} \quad=$ the third cumulant

$\mathrm{N}=$ total asperities number

$\mathrm{P} \quad=$ load

$\mathrm{p}_{0} \quad=$ maximum contact pressure

$\mathrm{p}(\mathrm{x}) \quad=$ random variable of normal distribution

$\mathrm{R} \quad=$ radius of the curvature

$\mathrm{r} \quad=$ positional variable in $\mathrm{x}-\mathrm{y}$ plane

$\mathrm{s}_{\mathrm{k}} \quad=$ skewness

$\mathrm{W}=$ maximum existence probability in the maximum value

$\mathrm{z} \quad=$ height axis

$\mathrm{Z}_{1} \quad=$ height displacement of asperity 1

$\mathrm{z}_{2} \quad=$ height displacement of asperity 2

$\Gamma \quad=$ peak to peak height

$\Phi($ ) = probability density distribution

$\Psi \quad=$ plastic limit

$\beta=$ contact angle at the side contact

$\gamma=$ dimension factor

$\delta_{1}=$ height displacement of asperity 1 due to pressure $\mathrm{P}$

$\delta_{2} \quad=$ height displacement of asperity 2 due to pressure $\mathrm{P}$

$\eta \quad=$ asperity density

$\mu \quad=$ mean height

$v \quad=$ Poisson's ratio

$\sigma \quad=$ standard deviation

$\tau=$ coefficient of temperature rise due to the ratio of friction energy to real contact area

$\omega \quad=$ interference 


\section{Introduction}

The real contact area is a key parameter for analysing friction, wear, heat generation and contact resistance. Several contact theories to discuss the tribological properties have been proposed [1]. The real contact area is often evaluated using computational methods as it very challenging to obtain it experimentally. Pauli, one of the greatest scientists of the $20^{\text {th }}$ century, stated that "God made the bulk; surfaces were invented by the devil". This is true for not only physics, but also tribology. The real contact area determines the temperature rise at the sliding interface [2], and the temperature rise will affect the mechanical system performance. Kadiric et al. discussed the relationship between the temperature rise and the contact pressure distribution on the sliding rough surface [3]. The over-limit clearance of the sliding members, such as the piston ring, due to the thermal expansion from the friction heat could degrade the machine efficiency and cause a subsequent catastrophic breakdown. If the thermal effect induced by the friction heat can be included at the design stage of the powertrain, it would help in the manufacturing of more efficient engineering systems.

Phenomenon of friction heat has been investigated for years, however it is still poorly understood due to its measurement difficulties [4-7]. In fact, many researchers have tried to measure the real maximum temperature between sliding surfaces. Several temperature measurement methods, such as using an infrared thermometer [8,9], the embedded thermocouple method [10] and scrubbing materials that form a thermocouple such as ironconstantan [11-15], have been reported. Quinn [16] studied the "hot-spot" temperature and estimated the localised temperature rise over $\sim 700{ }^{\circ} \mathrm{C}$. Wang et al. [17] reported that the bulk surface temperature reached $\sim 200{ }^{\circ} \mathrm{C}$ for the transition of mild wear to severe wear in steel 52100.

The "flash temperature (hot-spot)" concept was proposed by Blok [4,5]. He studied the heat theory of the maximum temperature rise at the contact area with reference to the Peclet number for the steady state. Jaeger [6] formulated the average temperature rise in terms of the Peclet number based on the Bessel function. Archard reported the friction heat for various sliding conditions and used thermal equations to explain that the temperature rise is inversely proportional to the contact diameter [7]. Based on the hypothesis that the frictional energy induced due to sliding is a key parameter that affects the temperature rise, Yamamoto et al. introduced the friction energy measurement method for tribotesters [18-20]. The authors categorised and quantified friction energy into the five tribological phenomena, namely, wear energy, friction heat, strain energy, plastic deformation energy and chemical energy [21]. The results showed that more than $99 \%$ of the frictional energy turns into heat. It has been also proven that energy consumption due 
to wear at the interface is proportional to the frictional energy [20,22]. Fouvry [23] and Liskiewicz [24] have considered quantification of fretting wear based on dissipated energy.

Computer simulations are one of the promising methods to estimate the temperature rise. Aghdam and Khonsari [25] compared the friction temperature between simulations and experiments in reciprocating dry sliding. Bansal and Streator [26] used energy-partition rate parameter, to improve their numerical simulations, by incorporating sliding conditions, such as the sliding velocity and evolving material's properties. Yamamoto et al. [2] simulated the temperature rise on sliding surfaces between the DLC and a steel ball using the frictional energy and energy-partition rate. They verified the real contact area and the oxidised surface effect on the temperature rise on the sliding area.

As the real contact area of a sliding surface determines the average and localised temperature rise, precise real contact area data is highly desirable. As the sliding interface remains invisible, the real contact area can only be estimated by reliable contact theory. The micro-geometric texture, which results from random asperity array, makes the contact theory even more complex. The pioneering contact theory introduced by Greenwood and Williamson (GW model) was based on a statistical concept [27]. The authors assumed the asperity heights have a Gaussian distribution and estimated the real contact area and mean pressure by numerical calculations. The theory proved a proportional relationship between the normal load and contact area. The closed-form solution was novel at that time, though still limited, as it assumed that the asperities were of the same spherical shape and size, as opposed to a flat and rigid surface where the Hertzian contact theory could be applied. Since the asperity size has a Gaussian distribution, the tip size of each asperity should be varied with its height. The initial statistical solution has being improved by many researches, including Greenwood and Williamson. Bush, Gibson and Thomas extended the GW model using elliptical and parabolic asperities (BGT theory) [28]. Whitehouse and Archard [29] and Hisakado [30] modified the GW model by adding the nonuniform radii of curvature concept. Chang at el. proposed the elliptic elasticplastic microcontact model by introducing the effective radius ratio, $\gamma$ [31]. Lui et al. compared the contact areas derived from the Gaussian and exponential distribution of the asperity height using the effective radius ratio, $\gamma$ [32]. Furthermore, Greenwood embedded a term expressing the radius as the variable of the asperity height into his original formula to account for elliptical contacts [33].

Another approach for characterising the real contact area is derived on the premise that the surface roughness is composed of a group of sinusoidal curves. The advantage of the sinusoidal model is that the roughness data can be coupled with the Fast Fourier 
Transform (FFT) to analyse the surface roughness distribution. Providing that the surface topology is the synthesis of a Fourier series with a random phase, the statistical information can be described by the power spectrum. In his early work, Westaggard reported the exact closed-form solution of the contact model between the flat plane and the rough surface, on the basis that the asperity height varies as a pure sinusoidal curve of period L in the $\mathrm{x}$ direction [34]. Nayak developed the closed-form solution using an $\alpha$ parameter to indicate the bandwidth of the power spectrum density, namely, the range of the wavelength [35]. Johnson et al. used the exact displacement result based on the 2D and 3D sinusoidal pressure distribution written as a double Fourier series [36]. Stanley and Kato introduced the FFT method to improve the computational efficiency for the deterministic elastic model [37]. Jackson and Streator examined the iterative multiscale framework using the Fourier series coefficient obtained from FFT of the contact surfaces [38]. These sinusoidal models were constructed on the premise that the Hertzian contact theory is applicable to the sinusoidal curve.

The main limitation of existing contact theories for approximation of contact temperature is their static nature as opposed to the dynamic character of the frictional interface. However, discussing a new contact model regardless of its static or dynamic nature is of interest. If the contact theory based on the sinusoidal radius can be derived from the Hertzian theory, it can lead to the improved contact theory.

\section{Consideration of the real contact area: sinusoidal model}

The Hertzian theory has been applied to the contact problem on the premise that the tip of the asperity is spherical. However, the sinusoidal model can offer better representation of the asperity, as the curvature of the sinusoidal function is dependent of the ratio of the periodic length to the sinusoidal height. Hence, the contact equations based on the sinusoidal mode can resolve the real contact area according to the variation of the asperities' tip curvature. Therefore, we have considered how the Hertzian theory is applicable to the sinusoidal model by using the real contact area equation based on the modified Hertzian theory following the GW method.

\subsection{Contact mode}

Archard [39] noted that the asperity model of the rough surface can be described as a hierarchical system with "protuberance upon protuberance". There are several geometry levels to the contact model between the two bodies. Fig. 1 shows the geometrical concept for the two-body contact. It must be considered which level of the protuberance has the major contribution to the tribological process at the interface. 
The initial geometry category should be the inclination of the body surface (level 1 in Fig. 1). For considering the contact problem, it is assumed that the surfaces of the two bodies are parallel to each other. However, in reality, it is rare that the two surfaces are perfectly aligned, and the sphere contact that is independent of the parallel condition is exclusive. The next hierarchical level is waviness, as shown by level 2 in Fig. 1. Further down, the asperity scale is shown as level 3, and the subsequent levels are levels 4 and 5 described as micro-asperity and nano-asperity, respectively. It is important to understand which hierarchical level is dominating at the interface, however in general level 1 should be neglected. In the case where the wave range of level 2 is smaller than the height range of level 3 , it is not necessary to consider the waviness of the surface. Overall, the asperity dimension governs the contact problem, and Eq. (1) expresses the contact events, where $\Gamma$ stands for the peak-to-peak height for the level.

$$
\operatorname{MAX}(\Gamma(\text { Level 1), } \Gamma(\text { Level 2), } \Gamma(\text { Level 3), } \cdots)
$$

The equation (1) implies that the level with the highest value is dominant in the contact mode. The present roughness measurement systems can diminish the surface waviness using the cut-off function and the surface roughness data can be extracted. However, in the case where the $\Gamma$ (Level 2) is dominating, it might be insignificant to evaluate the contact problem using level 3. Generally, $\Gamma$ (Level 2) could be greater than $\Gamma$ (Level 3), unless the surface is artificially controlled. In the case where the surface roughness is composed of a series of frequent curvatures, the dominant contact mode could be defined by the power spectrum density of the Fourier analysis.

\subsection{Contact equations of the sinusoidal model}

The contact equations of the sinusoidal model in this study were evaluated without taking into account the plastic index. Archard noted that the elastic contact only occurs in stable sliding [7]. Therefore, we focused on the real contact area in the elastic region for the temperature rise during stable sliding. The cycle length is proportional to the height by multiplying by the dimension factor $\gamma$. Eq. (2) gives the 2D model and the 2D curve is shown in Fig. 2.

$$
\mathrm{h}=h_{1} \cos \frac{\pi}{\gamma h_{1}} x
$$

where $h_{1}$ is the asperity height. The sinusoidal curves at $\gamma=2, \gamma=1$ and $\gamma=4$ are drawn in 
Fig.2 (a), Fig. 2 (b) and Fig.2 (c), respectively. The curvatures of the sinusoidal curves are altered with the dimension factor $\gamma$.

Eq. (3) gives the 3D model:

$$
\mathrm{h}=h_{1} \cos \frac{\pi}{\gamma h_{1}} x \cos \frac{\pi}{\gamma h_{1}} y
$$

Fig. 3 shows the 3D surface model, where the heights of the $\mathrm{x}$ and $\mathrm{y}$ directions are symmetrical. The proposed sinusoidal asperity model is more convenient than the previous models, because the asperity height and the radius of the contact tip can be controlled by hand $\gamma$ parameters independently.

When the asperity tip in a 3D sinusoidal model is brought into contact with the opposing surface with a given force, the contact area has a circular shape resulting from the Hertzian contact based on the sphere model.

The pressure distribution within the contact area is the same as the Hertzian calculation and the displacement, $\delta$, due to pressure, P, is obtained by Eq. (4),

$$
\delta_{1}=\frac{3}{4} \frac{\left(1-v_{1}^{2}\right) P}{a^{3} E_{1}}\left(a^{2}-\frac{r^{2}}{2}\right)
$$

where E is Young's' modulus, a is contact radius, $v$ is Poisson's ratio and $r$ is positional variable in $\mathrm{x}-\mathrm{y}$ plane.

where the shrinkage $\omega$ is:

$$
\omega=\left(z_{1}+\delta_{1}\right)+\left(z_{2}+\delta_{2}\right)
$$

$\omega$ can be obtained with the following Hertzian theory derivation, as shown in Fig. 4. Curves 1 and 2 represent the cosine curve with heights $h_{1}$ and $h_{2}$, and the dimension factors $\gamma_{1}$ and $\gamma_{2}$, respectively. The displacement $z_{1}$ is described by Eq. (6):

$$
z_{1}=h_{1}\left(1-\cos \frac{\pi}{\gamma_{1} h_{1}} r\right)
$$

As $r$ is close to zero, Eq. (6) can be transformed using a Taylor series by ignoring the higher power index, as shown in Eq. (7): 


$$
z_{1}=\frac{\pi^{2}}{2 \gamma_{1}^{2} h_{1}} r^{2}-\frac{\pi^{4}}{24 \gamma_{1}^{4} h_{1}} r^{4}+O\left(r^{6}\right) \approx \frac{\pi^{2}}{2 \gamma_{1}^{2} h_{1}} r^{2}
$$

The displacement $\mathrm{z}_{2}$ is also transformed, as well as Eq. (7):

$$
z_{2} \approx \frac{\pi^{2}}{2 \gamma_{2}^{2} h_{2}} r^{2}
$$

Inserting Eqs. (4), (7) and (8) into Eq. (5) creates Eqs. (10) and (11) because the equality Eq. (9) $=0$ is true when the factor $r$ is independent.

$$
\begin{gathered}
\frac{3}{4} \frac{P}{a E^{\prime}}-\omega+r^{2}\left\{\frac{\pi^{2}}{2}\left(\frac{1}{\gamma_{1}^{2} h_{1}}+\frac{1}{\gamma_{2}^{2} h_{2}}\right)-\frac{3}{8} \frac{P}{a^{3} E^{\prime}}\right\}=0 \\
\omega=\frac{3}{4} \frac{P}{a E^{\prime}} \\
\frac{\pi^{2}}{2}\left(\frac{1}{\gamma_{1}^{2} h_{1}}+\frac{1}{\gamma_{2}^{2} h_{2}}\right)=\frac{3}{8} \frac{P}{a^{3} E^{\prime}} \\
\because \frac{1}{E^{\prime}}=\frac{1-v_{1}^{2}}{E_{1}}+\frac{1-v_{2}^{2}}{E_{2}}
\end{gathered}
$$

The formulas of the sinusoidal contact model were modified using the asperity height and the dimension factor, instead of the radius of the sphere in the Hertzian contact formula, as shown in Eqs. (12)-(14),

$$
\begin{gathered}
a^{3}=\frac{3}{4} \frac{\gamma_{1}^{2} \gamma_{2}^{2} h_{1} h_{2}}{\gamma_{1}^{2} h_{1}+\gamma_{2}^{2} h_{2}}\left(\frac{1}{E^{\prime}}\right) \frac{P}{\pi^{2}} \\
\omega^{3}=\frac{9}{16} \frac{\gamma_{1}^{2} h_{1}+\gamma_{2}^{2} h_{2}}{\gamma_{1}^{2} \gamma_{2}^{2} h_{1} h_{2}}\left(\frac{1}{E^{\prime}}\right)^{2}(\pi P)^{2}
\end{gathered}
$$




$$
\begin{gathered}
p_{0}=k a=\frac{3}{2}(\pi P)^{\frac{1}{3}}\left(\frac{3}{4} \frac{\gamma_{1}^{2} \gamma_{2}^{2} h_{1} h_{2}}{\gamma_{1}^{2} h_{1}+\gamma_{2}^{2} h_{2}} \frac{1}{E^{\prime}}\right)^{-\frac{2}{3}} \\
\because \mathrm{k}=\frac{3 P}{2 \pi a^{3}} \quad \text { in Hertzian theory }
\end{gathered}
$$

In the case where the body 2 is a plane surface, $\gamma_{2} \mathrm{~h}_{2}$ is infinite. Hence, the equations can be modified as shown below:

$$
\begin{gathered}
a^{3}=\frac{3}{4} \frac{\gamma_{1}^{2}}{\pi^{2}} h_{1} \frac{1}{E^{\prime}} P \\
\omega^{3}=\frac{9}{16} \frac{\pi^{2}}{\gamma_{1}^{2}} \frac{1}{h_{1}}\left(\frac{1}{E^{\prime}}\right)^{2} P^{2} \\
p_{0}=k a=\frac{3}{2}(\pi P)^{\frac{1}{3}}\left(\frac{3}{4} \gamma_{1}^{2} h_{1} \frac{1}{E^{\prime}}\right)^{-\frac{2}{3}}
\end{gathered}
$$

Eqs. (12)-(14) were simplified on the basis that the dimension factor $\gamma$ is the same for both surfaces, as shown in Eqs. (18)-(20).

$$
\begin{gathered}
a^{3}=\frac{3}{4} \frac{\gamma^{2}}{\pi^{2}} \frac{h_{1} h_{2}}{h_{1}+h_{2}} \frac{1}{E^{\prime}} P \\
\omega^{3}=\frac{9}{16} \frac{\pi^{2}}{\gamma^{2}} \frac{h_{1}+h_{2}}{h_{1} h_{2}}\left(\frac{1}{E^{\prime}}\right)^{2} P^{2} \\
p_{0}=k a=\frac{3}{2 \pi}\left(\frac{\pi^{2}}{\gamma^{2}}\right)^{\frac{2}{3}} P^{\frac{1}{3}}\left(\frac{3}{4} \frac{h_{1} h_{2}}{h_{1}+h_{2}} \frac{1}{E^{\prime}}\right)^{-\frac{2}{3}}
\end{gathered}
$$

It is expected that the probability of contact of two opposing asperities at their tips is very low (Case I), and there is a much higher probability that the sides of the asperities are brought into contact (Case II), as shown in Fig. 5.

The formula of the radius of the curvature is Eq. (21): 


$$
\mathrm{R}=\frac{\left(1+f^{\prime}(a)^{2}\right)^{3 / 2}}{\left|f^{\prime \prime}(a)\right|}
$$

The radius of the curvature of Eq. (2) was obtained using Eq. (21):

$$
R_{1}=h_{1} \frac{\left(1+\frac{\pi^{2}}{\gamma^{2}} \sin ^{2} \frac{\pi}{\gamma h_{1}} x\right)^{3 / 2}}{\frac{\pi^{2}}{\gamma^{2}} \cos \frac{\pi}{\gamma h_{1}} x}
$$

In the case where $\mathrm{x}$ is nearly zero, Eq. (22) can be modified to Eq. (23) because $\sin \theta$ is equivalent to $\theta$.

$$
R_{1}=h_{1} \frac{\left(1+\frac{\pi^{4}}{\gamma^{4} h_{1}^{2}} x^{2}\right)^{3 / 2}}{\frac{\pi^{2}}{\gamma^{2}} \cos \frac{\pi}{\gamma h_{1}} x}
$$

If $\pi<\gamma$, Eq. (23) can be simplified, as shown in Eq. (24):

$$
R_{1}=\frac{h_{1}}{\frac{\pi^{2}}{\gamma^{2}} \cos \frac{\pi}{\gamma h_{1}} x}
$$

The cosine curve of Eq. (2) at position $\mathrm{x}$ is the equivalent to the radius $\mathrm{R}_{1}$ expressed by Eq. (24). The side wall contact area of the sinusoidal model was solved as the radius contact model based on Hertzian theory as shown in Fig.6. The Hertzian contact equation using the radius is shown in Eq. (25).

$$
a^{3}=\frac{3}{4} \frac{R_{1} R_{2}}{R_{1}+R_{2}} \frac{P}{E^{\prime}}
$$

Because the normal factor of the load $P$ at position $x$ is $P \cos \beta\left(\beta=\pi x / \gamma h_{1}\right)$, Eq. (25) can be recalculated using the radius Eq. (24), as shown in Eq. (26), which proves that the 
contact area resulting from bringing into contact the side walls of the asperities is the same as the contact area of the two tips of the asperities, as shown in Eq. (18).

$$
\frac{3}{4} \frac{\frac{h_{1}}{\frac{\pi}{2}^{2} \cos \beta} \cdot \frac{h_{2}}{\frac{\pi^{2}}{\gamma^{2}} \cos \beta}}{\frac{h_{1}}{\frac{\pi}{2}^{2} \cos \beta}+\frac{h_{2}}{\frac{\pi^{2}}{\gamma^{2}} \cos \beta}} \frac{1}{E^{\prime}} P \cos \beta=\frac{3}{4} \frac{\gamma^{2}}{\pi^{2}} \frac{h_{1} h_{2}}{h_{1}+h_{2}} \frac{1}{E^{\prime}} P=a^{3}
$$

\subsection{Real contact area based on the sinusoidal model}

The approximation of the Hertzian equations for an asperity were described by Timoshenco and Goodier [40] in the case where body 2 is a flat and rigid plane.

$$
a_{1}=R_{1}^{\frac{1}{2}} \omega_{1}^{\frac{1}{2}} \quad A_{1}=\pi R_{1} \omega_{1} \quad P_{1}=\frac{4}{3} E^{\prime} R_{1}^{\frac{1}{2}} \omega_{1}^{\frac{3}{2}}
$$

where $\omega_{1}$ is the interference $\left(h_{1}-d\right)$ for the asperity. The $h_{1}$ and $d$ are the heights of the asperity and the distance between the contact surfaces, respectively. The geometric relationship in an asperity is drawn in Fig. 7. One should realise that the shape of the asperities is characterised by a low angle slopes, though they are apt to be misunderstood as sharp peaks because the vertical scale of the roughness graph has a different scale.

The real contact area in the GW theory was derived based on Eq. (27). The contact theory of the sinusoidal model is obtained through the derivation of the $\mathrm{GW}$ equations analogically. Fig. 8 shows the geometric relationship between the flat and sinusoidal surfaces.

The difference between the asperity height and the interference $\omega$ is equal to the asperity height at the distance $\mathrm{a}_{1}$, as shown in Eq. (28). If $\mathrm{a}_{1}$ is small, it can be described as shown in Eq. (29) by the Taylor expansion of the cosine in Eq. (28).

$$
\begin{gathered}
h-\omega=h \cos \frac{\pi}{\gamma h} a_{1} \approx h\left(1-\frac{1}{2} \frac{\pi^{2}}{\gamma^{2} h^{2}} a_{1}^{2}\right) \\
a_{1}=\sqrt{2} \frac{\gamma}{\pi} h^{\frac{1}{2}} \omega^{\frac{1}{2}}
\end{gathered}
$$

The contact area $\mathrm{A}_{1}$ is: 


$$
A_{1}=\frac{2}{\pi} \Upsilon^{2} h \omega
$$

The contact load $\mathrm{P}_{1}$ is obtained from Eq. (16):

$$
P_{1}=\frac{4}{3} \frac{\gamma}{\pi} E^{\prime} h^{\frac{1}{2}} \omega^{\frac{3}{2}}
$$

The local pressure is calculated as:

$$
\frac{P_{1}}{A_{1}}=\frac{2}{3 \gamma} E^{\prime} h^{-\frac{1}{2}} \omega^{\frac{1}{2}}=\frac{2}{3 \gamma} E^{\prime} \sqrt{\frac{h-d}{h}}=\frac{2}{3 \gamma} E^{\prime} \sqrt{1-\frac{d}{h}}
$$

When the two surfaces contact until separated by a distance $d$, then any asperity which height was originally greater than $\mathrm{d}$ will be contacted. The probability of making contact over the given height $d$,

$$
\operatorname{prob}(h>d)=\int_{d}^{\infty} \Phi(h) d h
$$

$\Phi(\mathrm{x})$ is the probability density distribution.

Because $\omega=\mathrm{h}-\mathrm{d}$ and $\mathrm{A}_{1}=2 \gamma^{2} \pi^{-1} \mathrm{~h} \omega$, then the mean contact area is:

$$
\frac{2 \gamma^{2}}{\pi} \int_{d}^{\infty} h(h-d) \Phi(\mathrm{h}) \mathrm{dh}
$$

the expected real contact area $\mathrm{A}_{\mathrm{r}}$ is:

$$
A_{r}=\mathrm{N} \frac{2 \gamma^{2}}{\pi} \int_{d}^{\infty} h(h-d) \Phi(\mathrm{h}) \mathrm{dh}
$$

where $\mathrm{N}$ is the total asperity number in the apparent contact area $\mathrm{A}_{\mathrm{n}}$. Since $N=A_{n} \times \eta$, which is the asperity density, the real contact area rate is estimated by the following equation for the case where the asperity height has a Gaussian distribution. 


$$
\frac{A_{r}}{A_{n}}=\frac{2 \gamma^{2}}{\pi} \eta \int_{d}^{\infty} h(h-d) \frac{1}{\sqrt{2 \pi} \sigma} \exp \left(-\frac{1}{2}\left(\frac{h-\mu}{\sigma}\right)^{2}\right) d h
$$

where $\sigma$ and $\mu$ are the standard deviation and the mean height of the measured asperities on the rough surface, respectively. Normalisation by the variable transformation of $\mathrm{z}=$ $(\mathrm{h}-\mu) / \sigma$ makes Eq. (36), modified as below:

$$
\frac{A_{r}}{A_{n}}=\frac{2 \gamma^{2}}{\pi} \eta \int_{d_{0}}^{\infty}(\sigma z+\mu)\left(\sigma\left(z-d_{0}\right)\right) \frac{1}{\sqrt{2 \pi}} \exp \left(-\frac{z^{2}}{2}\right) d z
$$

where normalised $d_{0}$ is $(d-\mu) / \sigma$.

The expected total load $\mathrm{P}$ is expressed using Eq. (38).

$$
P=\frac{4}{3} \frac{\gamma}{\pi} \eta A_{n} E^{\prime} \int_{d}^{\infty} h^{\frac{1}{2}}(h-d)^{\frac{3}{2}} \phi(h) d h
$$

The normalised $\mathrm{P}$ by $\mathrm{A}_{n} \mathrm{E}^{\prime}$ is shown below:

$$
\frac{P}{A_{n} E^{\prime}}=\frac{4}{3} \frac{\gamma}{\pi} \eta \int_{d_{0}}^{\infty}(\sigma z+\mu)^{\frac{1}{2}}\left(\sigma\left(z-d_{0}\right)\right)^{\frac{3}{2}} \frac{1}{\sqrt{2 \pi}} \exp \left(-\frac{z^{2}}{2}\right) d z
$$

The current asperity model has been designed with the asperity width proportional to the asperity height by the dimension factor $\gamma$. The probability distribution of the asperity width is the same as the asperity height distribution multiplied by $\gamma$. Because the expected value of the asperity width distribution is a product of the mean height $\mu$ and $\gamma$, the asperity density is derived by the area $\left(1 \mathrm{~m}^{2}\right)$ divided by the average asperity area.

$$
\eta\left(/ m^{2}\right)=\frac{1}{\pi(\gamma \times \mu)^{2}}
$$

Eqs. (37) and (39) are modified by inserting the asperity density, Eq. (40). 


$$
\begin{aligned}
& \frac{A_{r}}{A_{n}}=\frac{2}{\pi^{2} \mu^{2}} \int_{d_{0}}^{\infty}(\sigma z+\mu)\left(\sigma\left(z-d_{0}\right)\right) \frac{1}{\sqrt{2 \pi}} \exp \left(-\frac{z^{2}}{2}\right) d z \\
& \frac{P}{A_{n} E^{\prime}}=\frac{4}{3 \pi^{2} \gamma \mu^{2}} \int_{d_{0}}^{\infty}(\sigma z+\mu)^{\frac{1}{2}}\left(\sigma\left(z-d_{0}\right)\right)^{\frac{3}{2}} \frac{1}{\sqrt{2 \pi}} \exp \left(-\frac{z^{2}}{2}\right) d z
\end{aligned}
$$

Eq. (41) indicates that the real contact area ratio is uniquely determined by the mean height $\mu$, standard deviation $\sigma$ and dimensionless $\mathrm{d}_{0}$ because of eliminating $\gamma$. Fig. 9 shows the relationship between the real contact area ratio and the normalised $\mathrm{d}_{0}$, calculated using Eq. (41) under the conditions of $\sigma=1.1 \times 10^{-8} \mathrm{~m}(0.011 \mu \mathrm{m})$ and $\mu=8.9 \times 10^{-8} \mathrm{~m}(0.089$ $\mu \mathrm{m})$. Fig. 10 shows the normalised pressure as a function of the normalised $\mathrm{d}_{0}$ with the same roughness condition as Fig. 9. The larger $\gamma$ is, which means the asperity is planarised, the smaller the normalised pressure is.

The real contact area ratio was plotted as a function of the normalised pressure using the common parameter $\mathrm{d}_{0}$ in Fig. 11 . The line obtained by the sinusoidal model is shifted to a lower pressure with increasing $\gamma$. The lines obtained by GW theory and predicted by Jackson and Streator (JS theory) [38] are also shown by the solid lines as references in the figure. The gradients obtained by the sinusoidal model and the reference lines are almost parallel. The line at $\gamma=5$ is overlapped with the GW line. The line at $\gamma=25$ is overlapped with the JS line. This proves the effectiveness of the sinusoidal model, in accordance with the references derived from elastic theory.

\section{Methodology}

\subsection{Materials}

DLC was deposited using radio frequency plasma enhanced chemical vapour deposition by feeding $15 \mathrm{sccm}$ benzene $\left(\mathrm{C}_{6} \mathrm{H}_{6}\right)$ at $0.3 \mathrm{~Pa}$ on a WC-9\% Co alloy. The thickness of the DLC was $\sim 1 \mu \mathrm{m}$. The nitrogenated DLC (denoted as NDLC) was produced by feeding the extra nitrogen flow rate at $30 \mathrm{sccm}$ [41]. The mechanical properties such as hardness, Young's modulus, mean roughness $\mu$, standard deviation $\sigma$ and skewness, of the specimens are listed in Table 1. The microhardness and Young's modulus of the DLCs were measured by a nano-indenter (Swiss CSM Instruments). A Berkovich stylus was penetrated into the DLC film, applying a maximum load at $5 \mathrm{mN}$ to keep the indentation depth less than $10 \%$ of the DLC thickness. An average of ten points of the hardness and Young's modulus was obtained. 
The 3D data of the surface height of the DLCs for pre- and post-tribotests were obtained by a laser microscope (OLYMPUS OLE-4000) with the resolution of $10 \mathrm{~nm}$.

Table 1. Hardness, Young's modulus and the roughness data of the DLCs.

\begin{tabular}{ccccccc}
\hline Specimen & $\begin{array}{c}\text { Hardness } \\
(\mathrm{GPa})\end{array}$ & $\begin{array}{c}\text { Young's } \\
\text { modulus } \\
(\mathrm{GPa})\end{array}$ & $\begin{array}{c}\text { Mean roughness } \\
(\mu \mathrm{m})\end{array}$ & $\begin{array}{c}\text { Standard } \\
\text { deviation }\end{array}$ & Skewness \\
\hline \multirow{2}{*}{ DLC } & Pre & \multirow{2}{*}{36.2} & 292 & 0.112 & 0.014 & 3.90 \\
& Post & & & 0.144 & 0.008 & 0.52 \\
\multirow{2}{*}{ NDLC } & Pre & \multirow{2}{*}{24.7} & 221 & 0.920 & 0.025 & 0.15 \\
& Post & & & 0.373 & 0.007 & -0.82 \\
\hline
\end{tabular}

\subsection{Tribological tests}

E52100 steel and alumina balls were used for the ball-on-disk test (RHESCA FRP-2100). The balls were cleaned ultrasonically in an acetone and ethanol baths for $3 \mathrm{~min}$ and dried at $50{ }^{\circ} \mathrm{C}$ for $30 \mathrm{~min}$. The tribotests of the DLCs against the balls were implemented immediately with conditions of the applied load $N$ of $9.8 \mathrm{~N}$, a sliding velocity of 100 $\mathrm{mm} / \mathrm{s}$ and a sliding distance of $200 \mathrm{~m}$ with lubricant-free conditions. The test environment was at room temperature $\left(\sim 20^{\circ} \mathrm{C}\right)$ and the humidity was $\sim 10-20 \%$. The friction energy $\boldsymbol{E}_{\mathrm{f}}$ was measured based on the $\boldsymbol{\Sigma} \boldsymbol{F}_{\boldsymbol{i}} \cdot \boldsymbol{S}_{\boldsymbol{i}}$ formula using the CSV data that logged the friction force at each sampling time in the ball-on-disk system [18-21].

The temperature rise on the sliding area between E52100 and the DLC was evaluated by the thermo-paint method (NiGK Corp.) at No. 13 (paint colour at $130{ }^{\circ} \mathrm{C}$ ), No. 26 (paint colour at $260{ }^{\circ} \mathrm{C}$ ), No. 33 (paint colour at $330{ }^{\circ} \mathrm{C}$ ) and No. 46 (paint colour at $460{ }^{\circ} \mathrm{C}$ ). The temperature indicator was painted on the steel ball before each tribotest. After the test, the paint colour near the sliding area was observed by the microscope to determine which paint No. turned the colour.

\subsection{Temperature distribution simulation}

The temperature distribution in the steel ball was simulated by ANSYS. The simulation model is shown in Fig. 12. The size of the 3D sphere model was $\phi 4.8 \mathrm{~mm}$ and the four diameters of the contact area were set at 60,90, 120 and $300 \mu \mathrm{m}$. The region near the contact area was divided into three layers of which the thicknesses were $0.01 \mathrm{~mm}$ for the first layer (contact layer), $0.09 \mathrm{~mm}$ for the second layer and $0.4 \mathrm{~mm}$ for the third layer in order to apply material properties, such as oxidised iron. The 3D model, of which the number of nodes and elements were 4870 and 1209, respectively, was simulated with 
different frictional energies obtained by the tribotests on the basis that the heat flow to the sliding direction is negligible [2]. Table 2 shows the initial parameters of the simulation for E52100.

Table 2. Initial conditions for the temperature distribution simulation of the E52100 ball.

\begin{tabular}{|c|c|c|c|c|}
\hline $\begin{array}{l}\text { Thermal } \\
\text { conductivity } \\
\mathrm{W} / \mathrm{m} \cdot{ }^{\circ} \mathrm{C}\end{array}$ & $\begin{array}{l}\text { Heat transfer } \\
\text { coefficient } \\
\mathrm{W} / \mathrm{m}^{2} \cdot{ }^{\circ} \mathrm{C}\end{array}$ & $\begin{array}{l}\text { Initial } \\
\left({ }^{\circ} \mathrm{C}\right)\end{array}$ & emperature & Frictional energy in watt \\
\hline \multirow[t]{4}{*}{46} & 4.65 & 22 & Layer 1 & $0.2 \quad$ (at $4.9 \mathrm{~N}$ test) \\
\hline & & 22 & Layer 2 & 0.4 (at $9.8 \mathrm{~N}$ test) \\
\hline & & 22 & Body & $0.8 \quad$ (at $19.6 \mathrm{~N}$ test) \\
\hline & & & & 1.2 (at $29.4 \mathrm{~N}$ test) \\
\hline
\end{tabular}

\section{Results and discussion}

\subsection{Temperature rise on the sliding surface}

Fig. 13 shows the cross section of the temperature distribution near the contact area for the steel ball. The maximum temperature rise is at the contact surface. The friction heat was dispersed rapidly into the ball and the temperature at $0.3 \mathrm{~mm}$ above the contact surface was near to the initial temperature. The maximum temperature rise as a function of the frictional energy and contact diameter is plotted in Fig. 14. The temperature rise becomes higher with increasing frictional energy or decreasing contact diameter.

Fig. 15 shows the temperature rise simulation results as a function of sliding velocity on the condition of the apparent contact diameter of the steel ball at $400 \mu \mathrm{m}$ against the DLC. For the calibration of the simulation data, the Peclet number at 0.8 of the DLC and the steel ball on the tribotest conditions was calculated using thermal conductibility, specific heat and sliding velocity. The energy partition rate of the contact materials at a Peclet number of 0.8 was estimated using the energy partition function [26]. The simulation results were calibrated by the obtained energy partition rate at $48 \%$ for the steel ball and at $52 \%$ for the DLC [2]. The temperature rises on the three-different real contact area ratio at $1 / 10,1 / 100$ and $1 / 1000$ are plotted. The temperature rise was higher with decreasing real contact area ratio. The determination of the real contact area ratio is important to estimate the temperature rise on the sliding surface because the temperature rise is very sensitive to the contact area.

Fig. 16(a) and (b) shows the thermal indicator results on the sliding area of the steel ball and the DLC, respectively. Fig. 16(a) is the optical image near the wear scar on the steel ball and Fig. 16(b) shows the wear track on DLC painted by the No. 26 thermal indicator 
after the tribotest at a sliding speed at $100 \mathrm{~mm} / \mathrm{sec}$ and the load at $9.8 \mathrm{~N}$. It was verified that the original light blue colour of the No. 26 turned to dark red on both specimens, which indicated that both sliding surfaces experienced over $260{ }^{\circ} \mathrm{C}$ but less than $330{ }^{\circ} \mathrm{C}$. The temperature rise approximately at $260{ }^{\circ} \mathrm{C}$ corresponding to the thermal indicator test at $100 \mathrm{~mm} / \mathrm{sec}$ was obtained on the 1/1000 line in the simulation result presented in Fig. 15.

\subsection{Comparison of the real contact area obtained from the experiments and simulation}

The real contact area ratio between the steel ball and the DLC on the present tribotest conditions was estimated approximately at $1 / 1000$ by the thermal indicator test and the computer simulation. The normalised pressure in this experiment, of which the measurement parameters were the contact diameter at $400 \mu \mathrm{m}$, the load at 9.8 N, Young's modulus of the steel at $210 \mathrm{GPa}$ and the DLC at $321 \mathrm{GPa}$, was $\sim 5 \times 10^{-4}$. The real contact area ratio at 1/1000 was obtained on the line of $\gamma=1$ in Fig. 11. This could mean the micro-asperity contact was dominant in the present sliding experiment because the underlying waviness or larger asperities could be characterised by a larger $\gamma$ parameter.

It is clear that the asperity dimension affects the contact area significantly. Further investigations could be required where $\gamma$ is not constant, but rather distributed, or the opposite body is not flat, but has different roughness.

\subsection{Evaluation of the real contact area ratio using the surface height distribution}

Counting the asperity number is difficult practically because the definition of the asperity shape is obscure due to the awkward shape. The definition using the three-point or sevenpoint method of the countable asperity is discussed [42]. The number of the asperities is also sensitive to the measurement resolution. In this section, the estimation method of the real contact area ratio based on the surface height distribution instead of the asperity height distribution is proposed. Fig. 17 shows the interference $\omega$ region between the rough and flat surface in two dimensions.

The two asperities in the interference are shown in Fig. 17. The areas in the region of the two asperities are also different, as indicated by the thick black lines, because both sizes are different. This means that the contact area of the asperities is equivalent to the asperity area located in the interference space and the number of the asperities is not significant. The laser microscope measures the surface height in each segment, for instance, the 1024 $\times 1024$ measurement segment is shown in Fig. 18. In other words, one data point obtained 
by the 3D laser is representative of the segment area. The integration of the normalised distribution for the surface height must be at 1 , which means the observation area is also normalised as 1 . When a rigid and flat plane is pressed against the rough surface until distance $\mathrm{d}$, the real contact area ratio to the apparent area can be estimated by the area of the integration of the normalised surface height distribution between the normalised $\mathrm{d}_{0}$ and the maximum asperity height $h$, as shown in Fig. 19. Plastically deformed area can be obtained by integration between the plastic limit $\Psi$ and the maximum asperity height $\mathrm{h}$ [43]. Eq. (41) and Fig. 9 show that the real contact area ratio is uniquely determined by the dimensionless separation $\mathrm{d}_{0}$ at the given mean height and standard deviation, which is equivalent to the method presented in this section. If the displacement $\mathrm{d}$ is caused by the given pressure, the real contact area ratio could be estimated using the surface height distribution parameters such as the mean height $\mu$ and standard deviation $\sigma$ obtained by $3 \mathrm{D}$ surface height data and the normalised $\mathrm{d}_{0}$. Hence, the real contact area during sliding can be estimated by the 3D surface height data obtained after sliding test.

Stated differently, if the mean height $\mu$, standard deviation $\sigma$ and the real contact area were known, the gap between the two surfaces could be estimated. The surface height distribution of both DLC coatings before and after test measured by the 3D laser microscope are shown in Figs. 20 and 21, respectively. The real contact area ratio of $1 / 1000$ was equal to the area of the normal distribution between $\mathrm{d}_{0}$ and $\mathrm{h}$. Therefore, the gap at the four interfaces listed in Table 1 against the rigid flat surface can be evaluated under zero skewness. The $\mathrm{d}_{0}$ at the area $=1 / 1000$ is approximately 3.1 as per a standard normal distribution tabulated values. Hence, the gap can be calculated using the formula $\mathrm{d}_{0}=(\mathrm{d}-\mu) / \sigma$ (Table 3$)$. One can notice, that the separation of surfaces after the tribological test was reduced. A precise knowledge of the extent of the separation would be a useful parameter for the development of sealing technology.

Table 3. Separation between the mean height of the rough surface and the flat surface $d-\mu$.

\begin{tabular}{lcccc}
\hline Surface & DLC pre- test & DLC post-test & NDLC pre- test & NDLC post-test \\
\hline Separation $(\mu \mathrm{m})$ & 0.043 & 0.025 & 0.078 & 0.022 \\
\hline
\end{tabular}

\subsection{Entropy of surface roughness}

The real contact area ratio during the sliding could be estimated using the method proposed in Section 4.3 by measuring the gap between the mean height and the opposite surface and the 3D surface roughness information.

Table 1 shows that skewness parameters after tribotests were decreased due to wear. The skewness is deviation index from the Gaussian distribution resulting from the height 
distribution deformed by wear or polishing operation. The skewness parameter $S_{k}$ is derived from the ratio of the third moment and the cube of the standard deviation:

$$
S_{k}=\frac{\frac{1}{n} \sum_{i=1}^{n}\left(x_{i}-\bar{x}\right)^{3}}{\left(\sqrt{\frac{1}{n-1} \sum_{i=1}^{n}\left(x_{i}-\bar{x}\right)^{2}}\right)^{3}}=\frac{m_{3}}{\sigma^{3}}
$$

It is assumed that the peaks of the asperities are being shaved off by the sliding, as shown in Fig. 22. The surface height distribution is then deviated to the left, as shown in Fig. 23, and the skewness is decreased based on the skewness definition of Eq. (43).

The entropy obtained from the probability theory proves that the probability density distribution with the maximum entropy is Gaussian in nature. It is worth noting that also Greenwood and Williamson introduced the asperity height distribution as a Gaussian distribution. Hence, the native roughness is characterised as the Gaussian distribution from an entropic point of view.

The entropy concept was established not only in thermodynamics and statistical mechanics, but also in probability theory, especially information processing theory. Both entropies, originated from physics and mathematics, show that the quantitative indication of the disorder though the derivation processes is different. The entropy derived from Boltzmann is $k \log W$, where $W$ stands for the maximum existence probability in the maximum existence number, which state is in equilibrium conditions. In other words, the entropy has the maximum value at the equilibrium condition. Therefore, one could observe that the manufacturing operation, which creates the order, is in fact an activity reducing the entropy by using available energy.

The entropy $\mathrm{H}(\mathrm{x})$ of the normal distribution in the probability theory is obtained as below.

$$
H(x)=-\sum p_{i} \log p_{i}
$$

When $\mathrm{p}_{\mathrm{i}}$ is the random variable of the normal distribution, $\mathrm{p}(\mathrm{x})$ is:

$$
p(x)=\frac{1}{\sqrt{2 \pi} \sigma} \exp \left(-\frac{1}{2}\left(\frac{x-\mu}{\sigma}\right)^{2}\right)
$$




$$
H(x)=-\int_{-\infty}^{\infty} p(x) \log p(x) d x=\log \sqrt{2 \pi e} \sigma
$$

Eq. (46) indicates that the entropy of the normal distribution is dependent on the standard deviation $\sigma$. We considered how the height distribution is varied by the wear or polishing. The standard deviation can be expressed by transforming Eq. (43):

$$
\sigma=\sqrt[3]{\frac{m_{3}}{s_{k}}}
$$

This predicts that the entropy is lowered by generation of the skewness from Eqs. (46) and (47).

\subsection{Thermodynamic approach to study the temperature rise}

There are several papers that discuss the tribological properties in terms of thermodynamics. Banjac et al. reported the temperature rise and wear from a thermodynamical point of view [44]. Bryant [45], Amiri and Konsari [46,47] employed entropy as a measure of the degradation caused due to wear and formation of dissipative structures during friction. Yamamoto introduced the thermodynamics equations based on the frictional energy [48].

During sliding, heat and wear are generated by the friction energy. Most of the frictional energy is wasted as heat [21] and the temperature due to the frictional energy becomes stable in the equilibrium state, in which it is assumed that the wear volume is minimal. Since the sliding region is a constant pressure, the Gibbs free energy is:

$$
\Delta G=\Delta U+\Delta P V+p \Delta V-\Delta T S-T \Delta S=\Delta U-T \Delta S
$$

In the case where the internal energy $U$ is regarded as the dispersive frictional energy Er, $\mathrm{Er}$ is negative. When $\Delta \mathrm{G}=0$, Eq. (48) is:

$$
0=-\Delta E_{f}-T \Delta S \quad \Delta S=-\frac{\Delta E_{f}}{T}
$$

Yamamoto et al. [2] proved that the temperature rise on the sliding condition is 
proportional to the friction energy $E_{f}$ and inversely proportional to the real contact diameter a.

$$
T=\tau \frac{E_{f}}{a}
$$

where $\tau$ is the proportional factor. Insert (50) into (49):

$$
\begin{aligned}
-\Delta S & =\frac{a}{\tau} \frac{\Delta E_{f}}{E_{f}} \\
-S+S_{c} & =\frac{a}{\tau} \operatorname{Iog} E_{f}
\end{aligned}
$$

where $S_{c}$ is the integration constant. Eq. (52) describes the inverse relationship between the real contact area and the frictional energy (heat energy) at a given entropy. The increase of the real contact area and the reduction of the temperature rise take a balance to drive the temperature stable in the sliding state from the entropic point of view.

\subsection{Summary}

The proposed methodology can be used for estimation of the real contact area during sliding, which can improve design processes of mechanical devices by more accurate temperature rise prediction due to interfacial friction.

The existing contact theories, reviewed in the Introduction section, employ the sphere contact model based on Hertzian theory. However, asperity models applied to the sphere are limited as the tip of the curvature is uniquely determined by the radius. In contrary, the proposed sinusoidal asperity model is more convenient because the asperity height and the radius of the contact tip can be controlled by $h$ and $\gamma$ parameters independently. The modified real contact area theory is universal, and expandable, while offering an equivalent precision to the existing theories.

\section{Conclusions}

The effect of real contact area on the temperature rise at the sliding interface between DLC coating and steel ball was discussed in this paper. The following conclusions can be drawn from this work: 
- The real contact theory based on the sinusoidal model was derived from the modified Hertzian theory and the measurement method of the real contact area was proposed. As the result, real contact area ratio is uniquely determined by the mean height $\mu$, standard deviation $\sigma$ obtained by $3 \mathrm{D}$ surface height distribution and the dimensionless $\mathrm{d}_{0}$.

- Good correlation between the modified contact theory based on sinusoidal model and Greenwood-Williamson model was demonstrated.

- The temperature rise at the DLC/steel sliding interface was estimated by the thermal indicator paint and it was found that the sliding area experienced temperature rise to over $260^{\circ} \mathrm{C}$.

- The real contact area to nominal contact area ratio of 1/1000 was estimated using the computer simulation.

- The real contact area and the temperature rise take a balance to drive the temperature stable in the sliding state at a given entropy.

\section{Acknowledgements}

The authors wish to thank Professor Hideo Honma (Kanto Gakuin University) for giving the opportunities to study this work in the laboratory and Dr. Ichimura for helpful discussions about this study.

\section{References}

[1] Jackson RL, Green I. In the modeling of elastic contact between rough surfaces. Tribol Trans 2011;54:300-14.

[2] Yamamoto S, Okuaki T, Egashira M, Kondoh K, Masuda C. Evaluation of the temperature distribution in steel balls induced by friction generated during the tribo-test against diamond-like carbon coatings. Tribology - Materials, Surfaces \& Interfaces 2015;9(1):33-40.

[3] Kadiric A, Sayles RS, Ioannides E. Thermo-mechanical model for moving layered rough surface contacts. J Tribol 2008;130:011016.

[4] Blok H. Theoretical study of temperature rise at surfaces of actual contact under oiliness lubricating conditions. Inst. Mech. Eng., Proc. General Discussion Lubrication and Lubricants 1937;2:222-35.

[5] Blok H. The flash temperature concept. Wear 1963;6:483-94.

[6] Jaeger JC. Moving Sources of heat and the temperature at sliding contacts. Proc Royal Soc New South Wales 1942;56:203-24.

[7] Archard JF. The temperature of rubbing surface. Wear 1958/59;2:438-55. 
[8] Bowden FP, Tabor D. The friction and lubrication of solids. London: Oxford Univ. Press; 1950.

[9] Shiozaki N, Harada M. Cutting edge temperature of face-milling cutter. Sci. Papers Inst. Phys. Chem. Res. 1962;56:209-15.

[10] Aghdam AB, Khonsari MM. Prediction of wear in reciprocating dry sliding via dissipated energy and temperature rise. Tribol Lett 2013;50,:365-78.

[11] Shore H. Thermoelectric measurement of cutting tool temperatures. J. Wash. Acad Sci $1925 ; 15: 85-8$.

[12] Bowden FP, Ridler KEW. Surface temperature of sliding metals. Cambridge Phil Sac Proc 1935;31:431-2.

[13] Furey MJ. Surface temperatures in sliding contact. ASLE Trans 1964;7:133-46.

[14] Dayson C. Surface temperatures at unlubricated sliding contacts. ASLE Trans 1967;10:169-74.

[15] Uetz H, Sommer K. Investigations of the effect of surface temperatures in sliding contact. Wear 1977;43:375-88.

[16] Quinn TFJ. The Effect of "Hot-Spot" Temperatures on the unlubricated wear of steel. ASLE trans 1967;10:158-68.

[17] Wang Y, Lei T, Yan M, Gao C. Wear rate, frictional temperature, and energy consumption of steel 52100 with different microstructures during sliding. J Phys D Appl Phys 1992,;25:A165.

[18] Yamamoto S, Kawana A, Masuda C. Tribological behaviour of stainless steel with respect to that of DLC in terms of energetic aspects. Tribology - Materials, Surfaces \& Interfaces 2013;7(4):161-7.

[19] Yamamoto S, Kawana A, Masuda C. Tribological behavior of diamond-like carbon produced by rf-PCVD based on energetic evaluation. Surf Coat. Technol 2013;236:45764.

[20] Yamamoto S, Egashira M, Kondoh K, Masuda C. Evaluation of the wear energy consumption of nitrogenated diamond-like carbon against alumina. Tribol Lett 2014;55:279-88.

[21] Yamamoto S, Egashira M, Kondoh K, Masuda C. Quantification of the wear, strain and heat energy consumption rates for sliding steel ball against diamond-like carbon coatings. Tribology - Materials, Surfaces \& Interfaces 2015;9(2):71-6.

[22] Yamamoto S. Physical meaning of the wear volume equation for nitrogenated diamond-like carbon based on energy consideration. Wear 2016;368-369:156-61.

[23] Fouvry S, Liskiewicz T, Kapsa P, Hannel S, Sauger E. An energy description of wear mechanisms and its applications to oscillating sliding contacts Wear 2003; 255:287-98. 
[24] Liskiewicz T, Fouvry S. Development of a friction energy capacity approach to predict the surface coatings endurance under complex oscillating sliding conditions. Tribol Int 2005;38:69-79.

[25] Aghdam A.B, Khonsari MM. Prediction of wear in reciprocating dry sliding via dissipated energy and temperature rise. Tribol Lett 2013;50:365-78

[26] Bansal DG, Streator JL. A method for obtaining the temperature partition at the interface of sliding bodies. Wear 2009;266:721-32

[27] Greenwood JA, Williamson JBP. Contact of nominally flat surfaces. Proc R Soc A 1966;295:300-19.

[28] Bush AW, Gibson RD, Thomas TR. The elastic contact of a rough surface. Wear 1975;3:87-111.

[29] Whitehouse DJ, Archard JF. The properties of random surface of significance in their contact. Proc R Soc A, 1970;316:97-121.

[30] Hisakado T. Effects of surface roughness on contact between solid surface. Wear 1974;28:217-34.

[31] Chang WR, Etsion I, Bogy DB. An elastic-plastic model for the contact of rough surface. J Tribol 1972;109:257-63.

[32] Liu Z, Neville A, Reuben RL. An analytical solution for elastic and elastic-plastic contact models. Tribol Trans 2000;43:627-34.

[33] Greenwood JA. A simplified elliptic model of rough surface contact. Wear 2006;261:191-200

[34] Westergaard HM. Bearing pressures and cracks. ASME J Appl Mech 1939;6: A49A53

[35] Nayak PR. Random process model of rough surfaces in plastic contact. Wear 1973;26:305-33.

[36] Jackson RL. An analytical solution to an Archard-Type fractal rough surface contact model. Tribol Trans 2010;53:543-53.

[37] Stanley HM, Kato T. FFT based method for rough surface contact. J Tribol 1997;119:481-85.

[38] Jackson RL, Streator JL. A multi-scale model for contact between rough surface. Wear 2006;261:1337-47.

[39] Archard JF. Elastic deformation and the laws of friction. Proc. R. Soc. A 1957;243:190-205.

[40] Timoshenco S, Goodier JN. Theory of elasticity. New York: McGraw-Hill; 1951 p. 372-382.

[41] Yamamoto S, Kawana A, Ichimura H, Masuda C. Relationship between tribological 
properties and $\mathrm{sp}^{3} / \mathrm{sp}^{2}$ structure of nitrogenated diamond-like carbon deposited by plasma CVD. Surf. Coat. Techn 2013;210:1-9.

[42] Pogacnic A, Kalin M. How to determine the number of asperity peaks, their radii and their heights for engineering surfaces: A Critical appraisal. Wear 2013;300:143-54.

[43] McCool JI. Comparison of models for the contact of rough surface. Wear 1986;107: 37-60.

[44] Banjac M, Vencl A, Otvic S. Friction and wear processes - thermodynamic approach. Tribology in Industry 2014;36:341-7.

[45] Bryant MD, Konsari MM, Ling FF. On the thermodynamics of degradation. Proc R Soc A 2008;464:2001-14

[46] Amiri M, Khonsari MM. On the thermodynamics of friction and wear- A Review. Entropy 2010;12:1021-49.

[47] Amiri M, Khonsari MM, Brahmeshwarker S. An application of dimensional analysis to entropy-wear relationship. J Tribol 2012;134:011604.

[48] Yamamoto S. Tribological mechanisms of steels with respects to diamond-like carbon in terms of energy input. Tribol Trans 2014;57:1001-6.

Figure captions

Figure 1. Contact geometries at three length scales.

Figure 2. 2D sinusoidal model: (a) $\gamma=2$, (b) $\gamma=1$, (c) $\gamma=4$.

Figure 3. 3D sinusoidal model.

Figure 4. Contact portion of the sinusoidal curve.

Figure 5. Contact mode between two asperities.

Figure 6. Side wall contact condition of the two asperities.

Figure 7. Surface roughness parameters.

Figure 8. Geometric relationship between the flat surface and sinusoidal asperity.

Figure 9. Relationship between the real contact area ratio and normalised $\mathrm{d}_{0}$.

Figure 10. Normalised pressure as a function of normalised $\mathrm{d}_{0}$.

Figure 11. Real contact area ratio plotted as a function of normalised pressure using $\mathrm{d}_{0}$.

Figure 12. Schematic of a ball model for the temperature simulation.

Figure 13. Temperature distribution in the steel ball.

Figure 14. Maximum temperature rise as a function of frictional energy and contact area.

Figure 15. Simulation results of the temperature rise at the sliding interface as a function of the sliding velocity and the real contact area ratio. 
Figure 16. Optical images of the thermal indicator paint near the worn area of (a) the steel ball and (b) DLC.

Figure 17. Contact area interference.

Figure 18. Measurement segment of the 3D laser microscope.

Figure 19. Real contact area ratio using the normalised surface height distribution.

Figure 20. Surface height distribution of the DLC on WC-9\%Co. (a) before tribotest, (b) after tribotest

Figure 21. Surface height distribution of the nitrogenated DLC on WC-9\%Co.

(a) before tribotest, (b) after tribotest

Figure 22. Polished asperities due to tribological interaction

Figure 23. Deviation of surface height distribution due to polishing.

Level 1

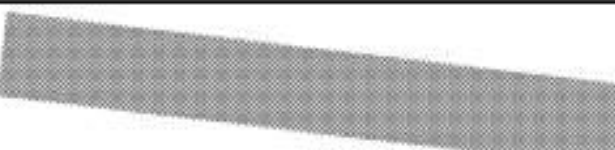

Level 2

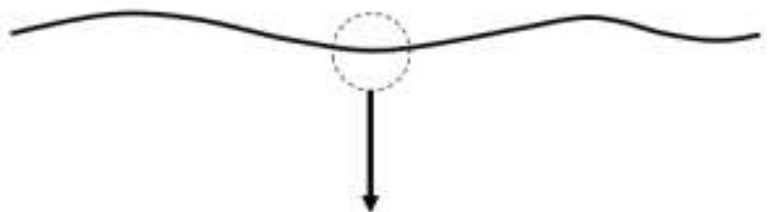

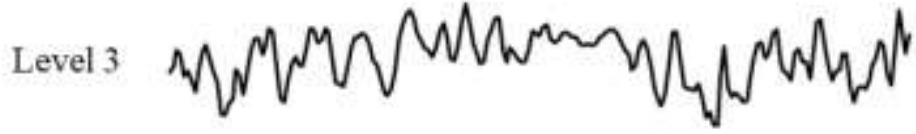

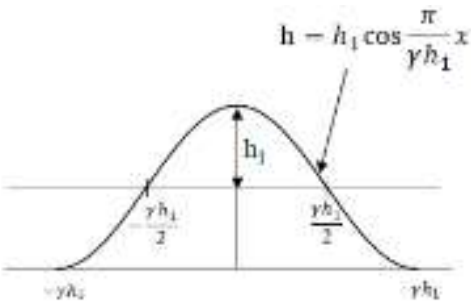



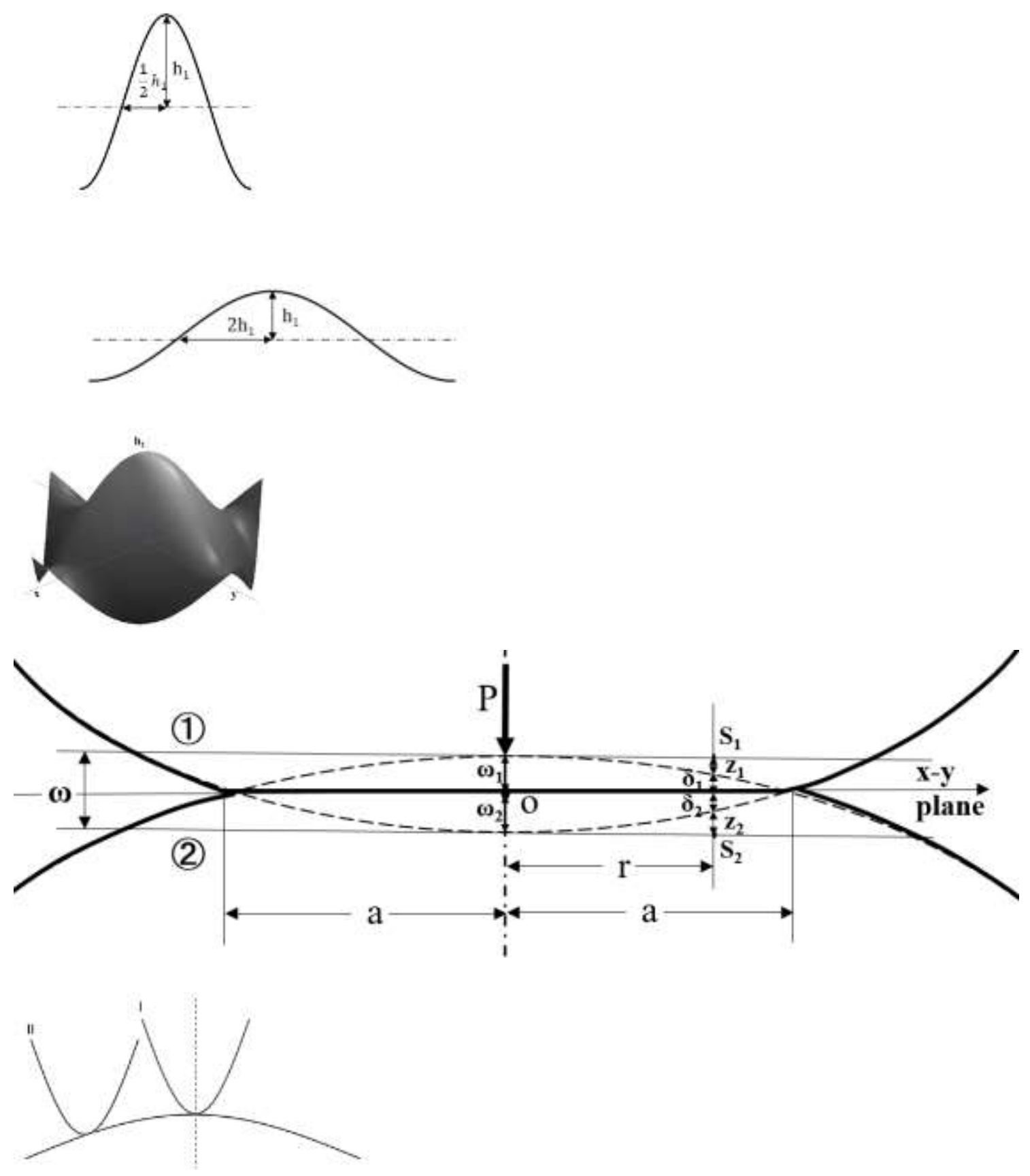

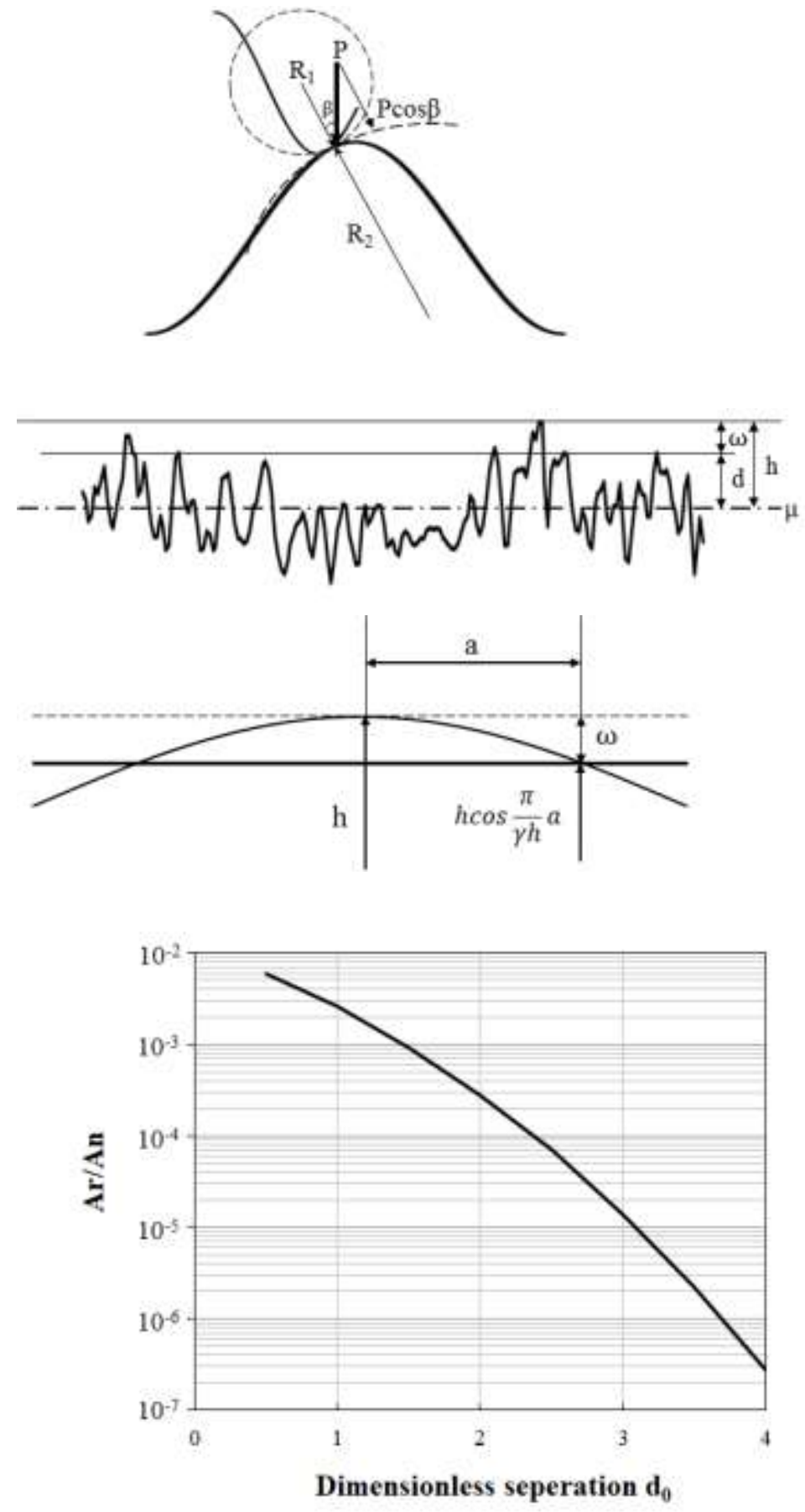

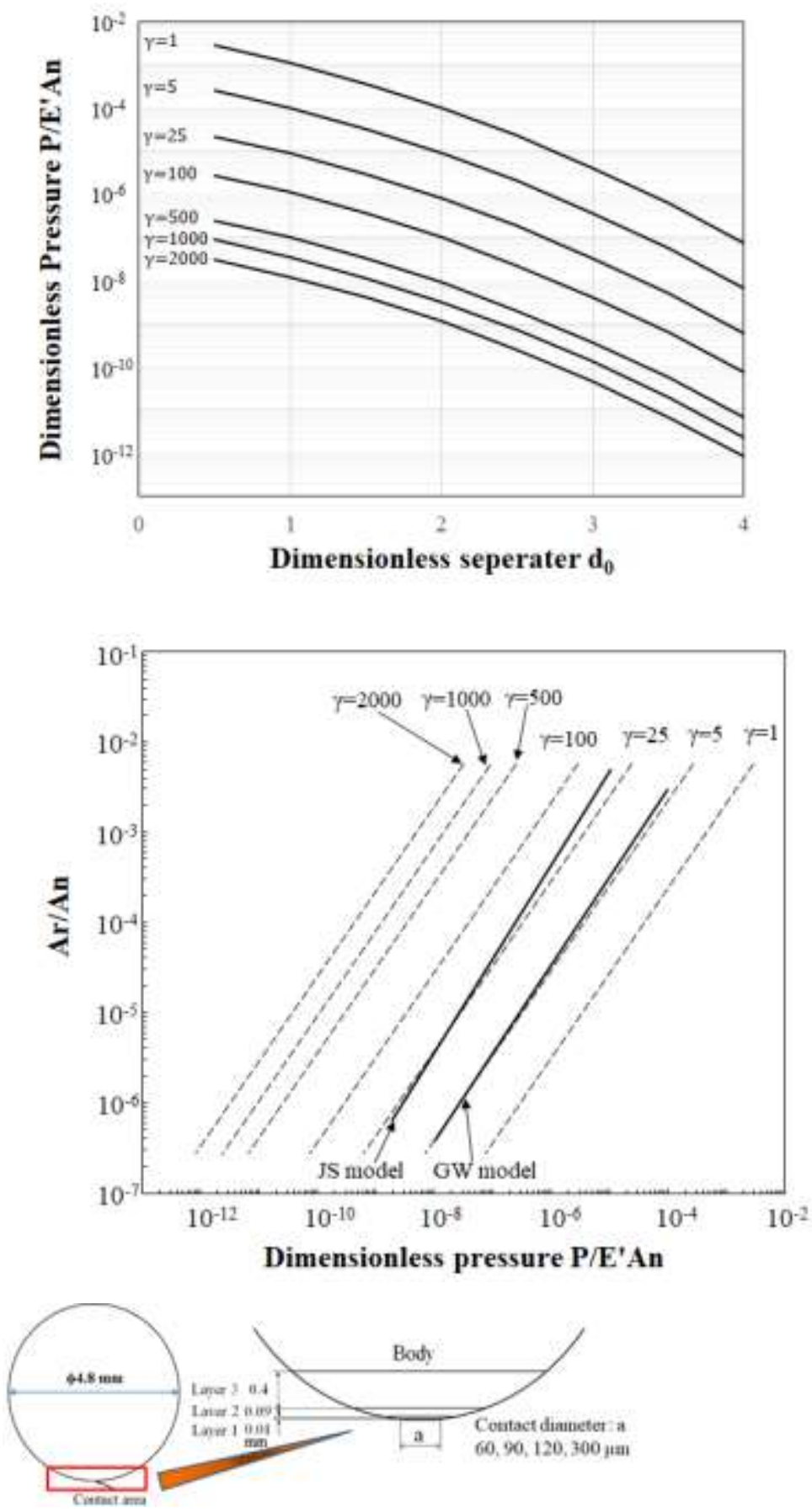

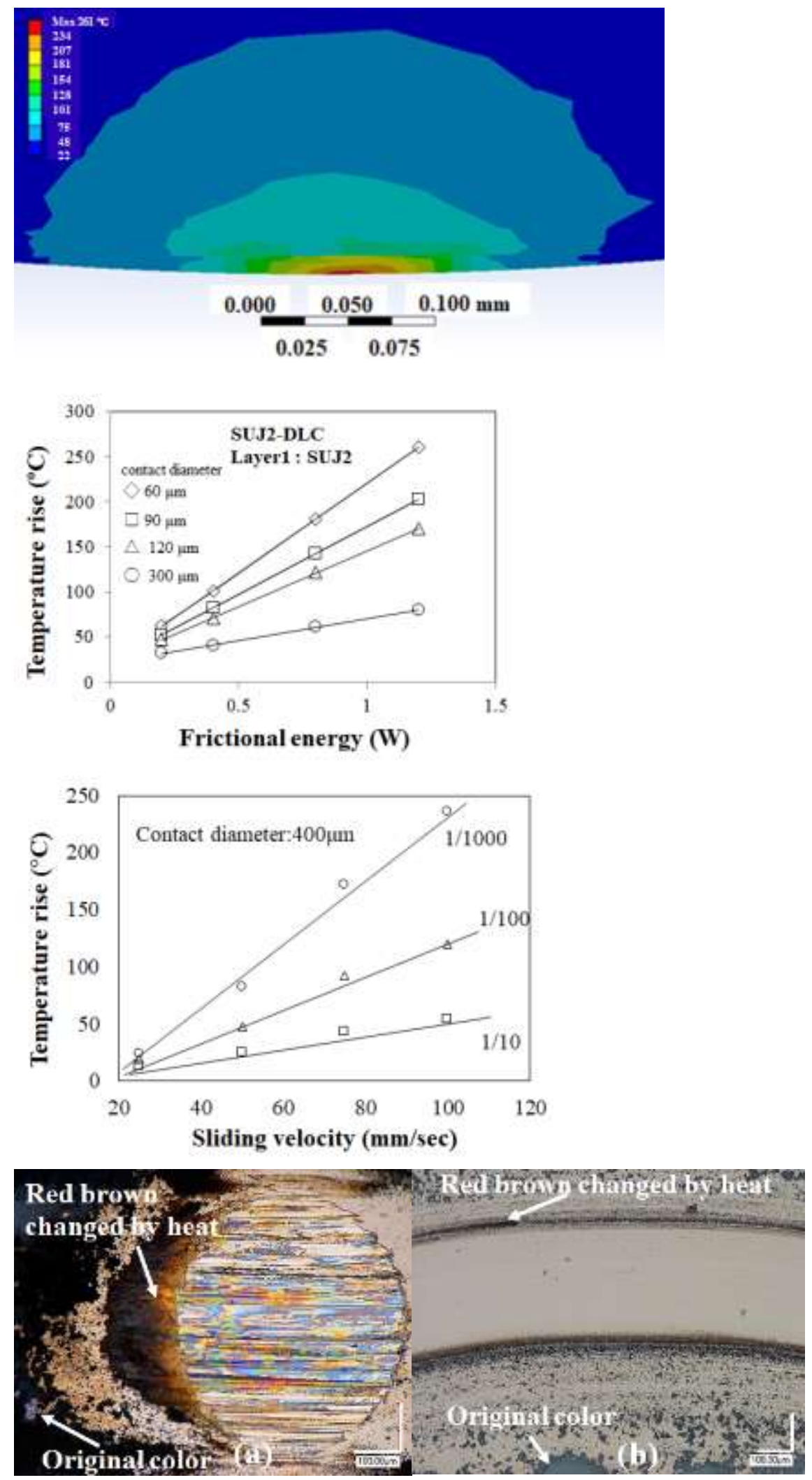

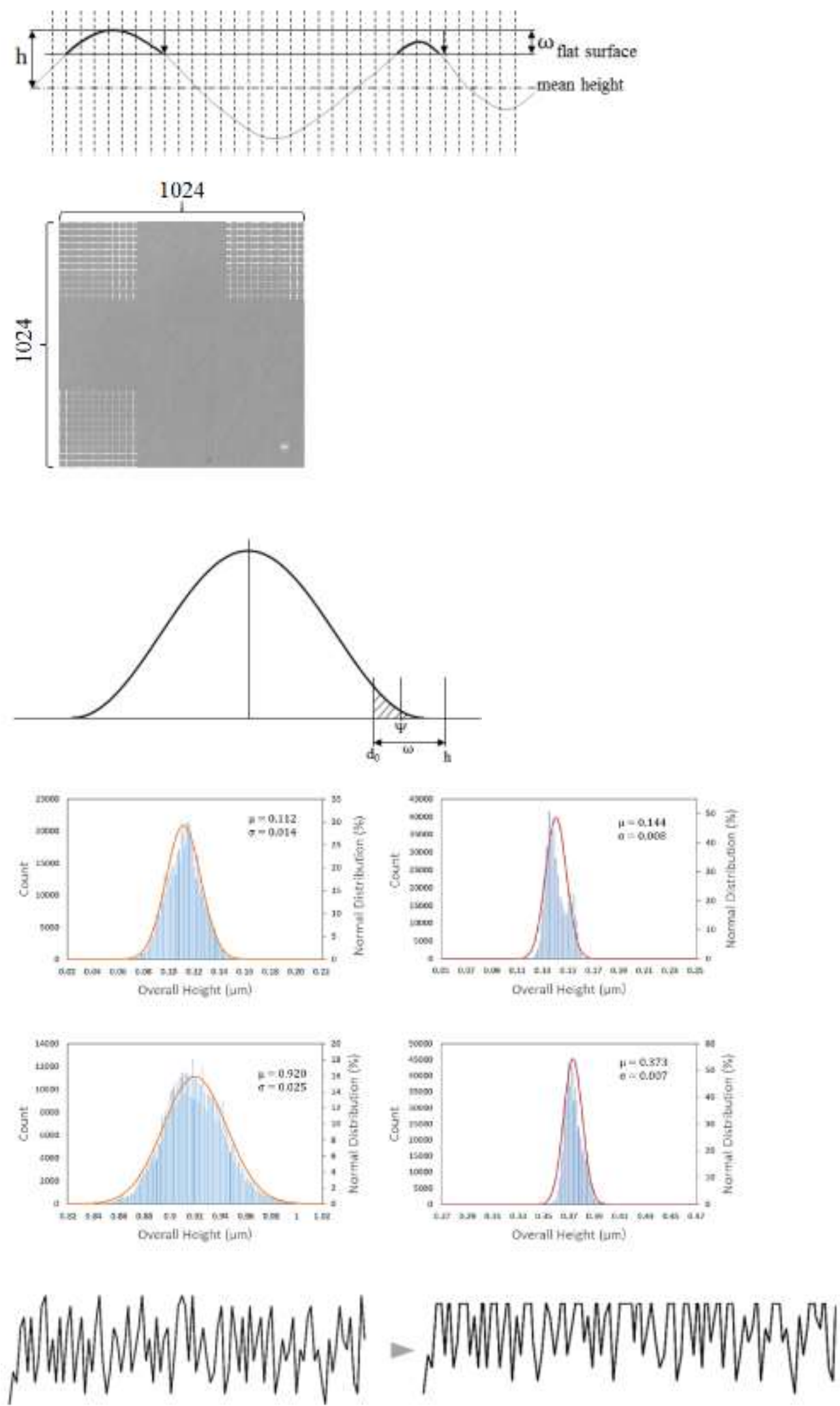


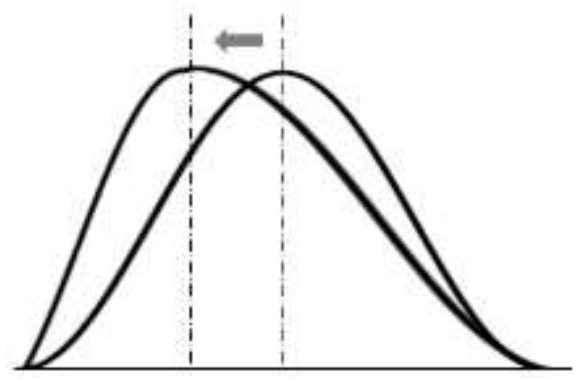

